\title{
A Framework for Access Control Models
}

\begin{abstract}
Burkhard Lau ${ }^{1}$
Summary

The goal of our framework for access control models, here described, is to provide a means to describe access control models in a uniform and consistent manner. Based on a thorough analysis of what access control means, three basic issues that an access control model should consist of are identified: entities, verification, and authorization. The latter two issues are discussed in detail and basic structures are identified again.
\end{abstract}

\section{Motivation}

The state of the art in access controls is dominated by two access control policies: Discretionary and Mandatory Access Control (DAC and MAC). What we need, however, is a more sophisticated view of policies than provided by the traditional black and white distinction between mandatory and discretionary controls due to the following main reasons:

It becomes more and more important that the access control model meets the requirements of a particular organization. So, an access control designer does not want to adapt the organization's requirement to fit into an existing (conventional) access control model. (S)he wants to describe a model fitting to the organization's requirements.

The integration of existing (database) systems in a distributed and heterogeneous (database) system includes the integration of access controls as well. To cope with this problem, a generalized model is necessary.

The solution is a framework which is able to describe existing access control models in a uniform and consistent manner by defining the fundamental parts that a model should consist of. Based on fundamental primitives, new models can be created as well. And our framework provides another advantage:

Different models can be described in terms of our framework which allows a comparison. In such a way, we are able to determine the main difference between DAC and MAC which is, as we will show, based on different paradigms.

Note that our approach is conform to the ISO framework for access control [ISO91]. We will, however, provide a much deeper insight into the basic structures of access control models.

We will proceed as follows:

After an overview and a delimitation, we describe the verification and administration aspect including paradigms in the chapters Three, Four, and Five. Chapter Six summarizes the framework.

Due to lack of space, we will only briefly show the application of this framework by describing well known access control models in terms of our framework. For a more comprehensive description as well as a comparsion with the Generalized Framework of Access Control (GFAC) by Abrams et.al [AbSm91, AbO192], we refer to the Ph.D. thesis [Lau95b].

\begin{tabular}{llll}
\hline & P.O. Box 356, NL-2600 AJ Delft \\
$+31-(0) 15-786328$ & Q & $\begin{array}{l}\text { B.Lau@IS.TWI.TUDelft.NL } \\
+31-(0) 15-786632\end{array}$
\end{tabular}




\section{CoAl of Access Control}

As the name indicates, access control controls accesses within an (information) system which we call in the following a target system. This characterization, however, is too broad, because it allows a very broad interpretation range including other countermeasures like integrity control. So, we would like to refine this view and to specify the goal of our investigations.

\subsection{OVERViEW}

Access control consists of two main parts [ISO91]: the Access control Enforcement Facility (AEF) and the Access control Decision Facility (ADF). The goals of these parts can be described as follows:

\section{Access control Enforcement Facility}

The AEF has to enfoce that only allowed activities can happen in the target system. Note that we do not aim at a special target system; it could be of any kind.

Access control Decision Facility

The ADF decides whether a particular access is authorized or not.

These two parts are related by the mapping activity $\leftrightarrow$ access and depend on each other. The AEF needs the decision of the $\mathrm{ADF}$, while the $\mathrm{ADF}$ needs the $\mathrm{AEF}$ to enforce its decision.

While the decision itself can be made independently on the target system, the enforcement has to be built into the target system. As described and motivated in [Lau95a], we consider these two facilities separately, assume an existing $\mathrm{AEF}$, and discuss in the following only the $\mathrm{ADF}$.

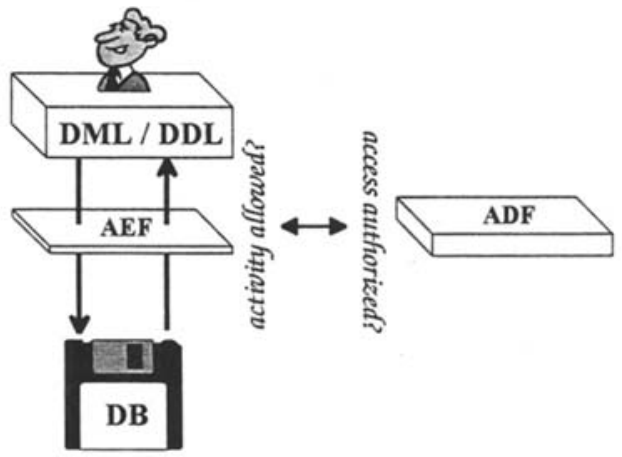

As we will not consider the $\mathrm{AEF}$ in the following, we make some comments here:

- There could be various access levels within a target system, proving different access functionality. Each level could require its own AEF. Here, however, we assume just one AEF and refer for further considerations to [Lau95b].

The AEF has, preferably, to be planed during the design of the target system. Attempts to stuff the AEF into a target system afterwards result often in a vulnerable system, because the system seems to be secure but the AEF can be bypassed.

A serious problem is to prevent bypassing the AEF. A possible solution is to encode the database and only to decode if the AEF is passed successfully. This, however, is not the complete solution, just an idea which requires further research.

As the AEF intercepts every activity anyway, it can write a record about every activity as well. So, the AEF can support auditing. 


\subsection{Interplay Between AEF and ADF}

While the AEF deals with activities in the target system, the ADF handles accesses. For a formal consideration, we need their definitions:

Definition 1 Activity

As we do not aim at a special target system, we define an activity as an abstraction of a couple of properties:

- The activity elements are part of the target system. Our understanding of a target system includes, consequently, users as well.

- The elements of an activity depend heavily on the kind of target system. Each activity, however, has an initiator, a target, and an activity mode which corresponds with the target.

- The activity threatens the security of the target system. Other activities can proceed without any checks. Note that the target system determines which security aspects (confidentiality, integrity, and/or availability) are concerned.

So, the AEF can check two aspects: is it an activity at all (Are the elements part of the target system? Corresponds the action with the object type?), and is it an activity which might threaten the security (Is, for example, the action dangerous or not?)? In the last case, the ADF has to be involved. It makes decisions about generalized activities called accesses, which makes it independent on the target system:

Definition 2 Access

A combination of one subject entity, action entity, and object entity is called access. So, an access $a^{2}$ is a triple $(s, a, o)$.

We denote the set of all accesses with $2 \mathbb{l}$, so $2 \mathbb{1}=\mathrm{S} \times \mathrm{A} \times \mathrm{O}$. Instead of the pair (action, object), also capabilities can be used ensuring that the action is applicable to the object. In this case, an access is abbreviated by the pair (subject, capability), denoted as $\mathfrak{a}=(\mathbf{s}, \mathfrak{c})$.

So, we need a mapping between activities and accesses. Dependent Entities of the target system on the activities, one element of the target system can act in different roles. In the one situation, it could be a subject and in another an object. Vice versa, if an action comprises a set of target system elements, this set will be mapped on just one object entity of the ADF. Summing up, it is a N:M mapping between the elements of the target system and the entities in the sense of access control.

Note that the Clark-Wilson approach [ClWi87] as well as polyinstantiation [JaSa90, JaSa91] deals precisely with this topic. While Clark and Wilson assign well-formed transactions to objects with the only action Execute, polyinstantiated relations allow security views on relational databases.

\section{Files Transactions \\ Objects User Programs \\ Relations Tuples}

Processes

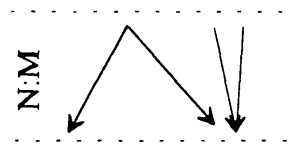

Subjects Actions Objects

Entities of the $\mathscr{A D F}$

Each access control entity will have a couple of properties. An object, for example, can have an owner, and a subject a particular clearance. Properties are essential to describe authorized accesses. They can have three sources: 
The Real World

As the target system is a mapping of the real world, a target system entity has got some properties of its origin. So, an entity in a car database gets properties of real cars, e.g. the age or the colour.

The Target System

The target system can add artificial properties as record number, identifier, or pointer.

The Access Control System

Access control could also require some properties, for example a security label.

Properties coming from the real world or from the target system are usually stored in the target system. There, access control attributes can be stored as well. It is, however, an implementation decision, how closely the databases of the target system and the ADF are connected.

\subsection{Coal of ADF}

The $\mathrm{ADF}$ has to answer the question:

\section{Who is (not) authorized to access what and how?}

Consequently, the ADF has to verify whether a particular access is authorized or not. Due to a formal consideration, we define:

\section{Definition 3 Access Status}

In accordance with Rabitti et.al., we call the set of all authorized accesses access status is therefore a subset of $2 \mathbb{A}$, so $\mathfrak{A} \subseteq 2 \mathbb{a}$. $\mathfrak{a} \in \mathbb{A}$ means consequently that $\mathfrak{a}$ is authorized.

can be filled by several sources.

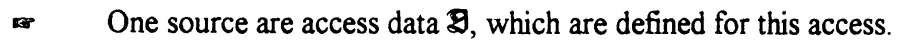

- Another source is described by inference control. Here, an access history is involved.

- Also the integrity of an access could be a reason to include or exclude an access.

As described in [Lau95a], it is meaningful to distinguish between several logical security modules. So, integrity control, inference control, and access control are in a logical sense separate modules with independent decisions. In the following, we will understand the goal of $\mathrm{ADF}$ as the filling of by only access data and nothing else. So, the goal of the ADF is to provide for a decision algorithm:

$$
\mathfrak{g} \rightarrow \mathfrak{B}
$$

Note that there is a subtle difference between accesses which are authorized ( $)$ and accesses

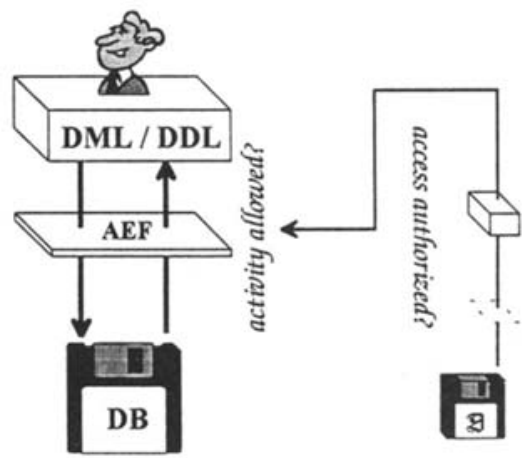
which should be authorized (\&). The art of authorization will be to identify these two sets. 


\subsection{Securing the ADF}

Of course, besides rare cases of statical ADF databases, the data of the $\mathrm{ADF}$ has to be administrated as well. In this sense, the ADF will be considered as a target system and we need an authorization control. Activities manipulating the ADF database have to be intercepted by an authorization control enforcement facility (ACEF), which cooperate with an authorization control enforcement facility (ACDF). This second ACDF has thus to answer the question:

Who is (not) authorized to authorize to access what and how?

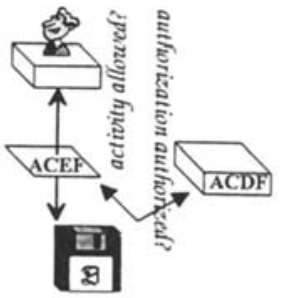

Here, we cope with the same problems as described up to here, again. We need an interception of the activities concerned with $\mathbb{Q}$, their mapping onto authorizations, their verification, and, finally, an administration of corresponding data $\%$. This data has to be protected again, and in such a way, we repeat ourselves. To find an end of this spiral, there has to be initial data $(\mathbb{Z}$, ...), which will be called a paradigm.

\subsection{Summary and Conclusion}

Summing up, access control consists of four major parts:

The realization of an AEF, which has, at least, to intercept every activity within a target system and has to involve the ADF.

The realization of an ADF, which has to be able to decide about every access whether this access is authorized or not.

A bijective mapping between activities and accesses to enable a communication between these two components.

The enforcement of administration rules which protect the ADF database.

An access control model, on the other hand, need not to cope with the AEF, but assumes merely an existing one. However, the other three aspects have to be described by such a model, because otherwise the description is not comprehensive.

We will give here a framework for access control models. So, we do not consider a special target system. That's why, we will not discuss the mapping onto access control entities, but assume an existing one. Note, however, that a particular access control model has to deal with it.

So, two aspects remain: verification and administration, which will be discussed now.

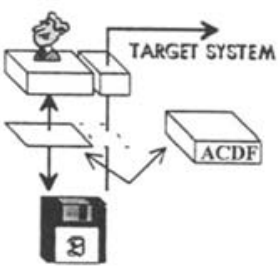

\section{The Verification $\mathfrak{Z} \rightarrow \mathbb{H}$}

Based on access data, the ADF decides whether an access is authorized or not, formal $\mathfrak{a} \in \mathbb{A}$ ? 


\subsection{Overview}

We will trace the verification process backwards, and show at each step, what alternatives exist. In an overview, we will investigate the following steps (from right to left):

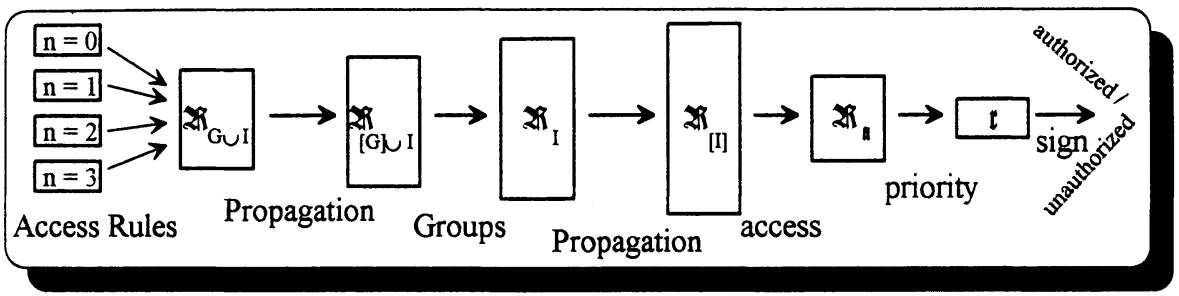

Figure 1 Overview of the Verification Algorithm

Roughly speaking, we distinguish between three major steps with various substeps producing intermediate right sets. Their extents are illustrated by the box size.

- Initial Rights $\mathbb{R}_{\mathrm{GuI}}$

Access rules of four types create a certain amount of initial rights for groups $G$ as well as for individuals I.

- Access Rights $2 \pi$

Based on $\boldsymbol{R}_{\mathrm{Gu}}$, propagation rules produce access rights $2 \mathbb{R}$ in three substeps: propagate between groups, resolve group memberships, and propagate between (individual) entities. Here, the transitive closure of rights on the group's level (G $\rightarrow[G])$ and on the individual's level $(\mathrm{I} \rightarrow[\mathrm{I}])$ is formed. The final transitive closure $\mathbb{R}_{[1]}$ are access rights what we denote with just $2 \mathbb{R}$ as well.

* Final Decision

After applying these access data, the access rights with the requested access are selected. From these access rights, the right with the highest priority is chosen. The sign of this right is decisive for the verification result.

Access data exist in three concepts, where the entity properties play an important role:

- Entities can be assembled in authorization groups to ease the administration. If groups are formed by a rule, groups are a means of abstraction.

Entity or group references can be exploited by propagation rules to decrease the administration effort.

Access rights can be derived from entity or group properties using access rules.

\subsection{ZI $\rightarrow$ AUThORIZEd / UNAUThORIZEd}

First of all, we want to describe the final decision whether an access is authorized or not.

\subsection{1 $t \rightarrow$ authorized / unauthorized: Applying the Sign}

There are positive, negative, and mixed ADF's describing permissions, prohibitions, or both. In mixed systems there is a need to characterize explicitly an access as a permission or a prohibition by a sign \pm , which is not necessary in positive or negative systems where signs are implicit. This leads to the notation of an access right $\mathfrak{t}$ as $\mathfrak{t}=(\mathfrak{a}, \pm)$. 
On the other hand, a closure assumption is needed for the case that there is no access right $t$ for the requested access $\mathfrak{a}$.

Not all combinations between system kind and closure assumption are possible. A combination of a negative $\mathrm{ADF}$ (describing just prohibitions) with the closed world assumption (everything is forbidden except explicitly given permission) is obviously meaningless. Assuming that the reader is familiar with the notations, we show the meaningful relationships:

\begin{tabular}{|cccc|}
\hline open world assumption & & & closed world assumption \\
$\boldsymbol{\uparrow}$ & $\boldsymbol{\pi}$ & $\boldsymbol{\pi}$ & $\boldsymbol{\uparrow}$ \\
negative ADF & mixed ADF & positive ADF \\
\hline
\end{tabular}

Figure 2 Meaningful Combinations Between System Kinds and Closure Assumptions

We can formally describe this step with:

$\exists \mathfrak{\imath}$ ? no: decision according to the closure assumption

yes: $\exists \pm$ ? no: decision according to the system kind

yes: decision according to the sign \pm which requires an interpretation

Note that there are also approaches to extend permissions and prohibitions by freedoms and duties. See, for a more comprehensive discussion, Morris and McDermid [MoDe92] or Lubinski [Lubi92, Lubi93].

\subsection{2 $2 \pi_{t} \rightarrow t:$ Applying Priorities}

In general, there are several access rights containing the same access, which we denote with 21. If there are different signs, there is the danger of conflicts: a conflict is given if there are two access rights $\mathfrak{t}^{i}$ and $\mathfrak{t}$ containing the same access $\mathfrak{a}$, but having a different sign:

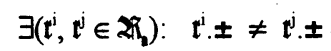

Priorities are introduced to avoid those conflicts, and so an access right becomes $\mathfrak{t}=(\mathfrak{a},( \pm, \mathfrak{p}))$ (see also [RaWK89, RBKW91]). Note that a priority $\boldsymbol{p}$ can be complex.

\subsubsection{Consistency Condition}

Two conditions on priorities are obvious:

- The priorities of two rights $\left(\mathfrak{t}^{\mathrm{i}}\right.$ and $\left.\mathfrak{t}^{j}\right)$ with the same access $\mathfrak{a}$ have always to be comparable, i.e., it has to be possible to determine the higher priority for each priority pair.

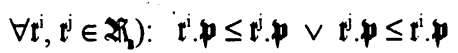

Consequently, we have to ensure a linear ordering between the priorities of a conflicting set of rights 2 .

2 Furthermore, in order to keep it possible to make a decision, we demand that every priority has to be unique. (We could accept $\mathfrak{t}^{\mathrm{i}} \cdot \boldsymbol{p}=\mathfrak{t}^{\mathrm{j}} \cdot \boldsymbol{p}$ if there is a third right $\mathrm{t}^{\mathrm{k}}$ with a priority $t^{k} \cdot$ which is higher than $t^{i} \cdot p$ and $t$ [Brüg92]. However, we have a problem when $t^{k}$ disappears and that is why we avoid this situation.)

$$
\forall\left(\mathfrak{t}^{\mathrm{i}}, \mathfrak{t} \in \mathbb{R}_{\mathfrak{i}}\right): \mathfrak{t}^{\mathrm{i}} \cdot \boldsymbol{p} \neq \mathfrak{t}^{\mathrm{j}} \cdot \boldsymbol{p}
$$


(1) and 2 are combined and form the following expression [Lau94]:

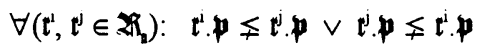

\subsubsection{Implicit Prionities}

Beside explicit priorities there is the possibility to have implicit ones. The basic idea is that priorities are deduced from parts of the access right. In particular:

Sign

A quite often used strategy is: a prohibition always takes priority over a permission. Note, however, that the idea of this proposal (in doubtful situations prefer denial of access) is controversial and violates an access control goal (to allow authorized accesses)! In critical situations the inability to perform an access can also cause damage.

Entity

Another strategy is to prefer the most specific right [Lunt89]: the right for an individual always takes priority over the right for a group. However, this, obviously, does not solve all conflicts. Beside this, we are in doubt whether it is possible to precisely define specificity at all (consider, e.g., unintended specificity or overlapping sets).

Here, the access right with the highest priority out of all rights concerned with a same access is selected.

\subsubsection{2i $\rightarrow$ 2i:: Applying Predicates}

Predicates are usually used to restrict the validity of an access to certain hours or by other logical expressions. They should be evaluated during this step.

\subsubsection{SUMmaRy}

We mentioned three possible attributes of an access: sign, priority, and predicate. While a sign is (even if implicitly) always present, an access control model has to determine which further attributes are used. In each case, we can define an access right as:

Definition 4 Access Right

An access right $\mathfrak{t}$ is a pair $\left(\mathfrak{a}, A_{s}\right)$ consisting of:

an access $\mathfrak{a}$ as defined above.

an attribute set $A_{s}$ which specifies the access.

An access control model has to define both $A_{s}$ and an algorithm to process $A_{s}$.

\section{$3.32 \pi_{G U I} \rightarrow 2 \pi$ III: Applying Propagation to Get Access Rights}

Initial rights $\mathbb{R}_{\mathrm{GuI}}$ are defined on access entities (S, A, and $\mathrm{O}$ ) as well as on authorization groups $\left(\mathrm{S}_{G}, A_{G}\right.$, and $\left.\mathrm{O}_{G}\right)$. So, $\boldsymbol{R}_{G \cup I} \subseteq 2 \mathbb{A}_{G \cup I} \times A_{S}$, with $2 \mathbb{A}_{G \cup I}=\left(S \cup S_{G}\right) \times\left(A \cup A_{G}\right) \times\left(O \cup O_{G}\right)$. On and between both levels, a propagation of rights can be defined. So, we distinguish between three sub-steps:

(In the following, $\mathfrak{a}_{E}$ denotes that $\mathfrak{a}=(\mathrm{s}, \mathrm{a}, \mathrm{o})$ contains certain elements $E$, so $E \subseteq\{\mathrm{s}, \mathrm{a}, 0\}$.)

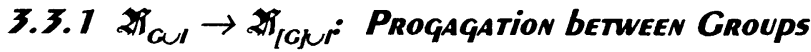

Relationships between groups can be exploited to propagate initial rights: 


\section{Facts}

The propagation structures are the facts. Consequently, the existence of entities in the concerned set and their mutual relationships have to be managed.

\section{Rules}

A propagation rule describes the propagation of rights between entities at an abstract level. The rule specifies the entity set and the direction of the relationship $\Re$ which can depend on the kind of access (prohibition or permission) or the kind of action (read or write), formally:

$$
\left(\mathfrak{a}_{\mathrm{g}}, \mathrm{A}_{\mathrm{s}}\right) \in \mathfrak{R}_{\mathrm{GUI}} \wedge\left(\mathrm{g}^{\prime}, \mathrm{g}\right) \in \Re \Rightarrow\left(\mathfrak{a}_{\mathrm{g}}, \mathrm{A}_{\mathrm{s}}\right) \in \mathfrak{R}_{\text {IGUI }}
$$

Note that one relationship can be exploited by several propagations. This is therefore a $1: \mathrm{N}$ relationship. Note furthermore that the transitive closure can only be achieved if the propagation rules are alternately applied.

\subsection{2 $2 \pi_{\text {IGUI }} \rightarrow 2 \pi_{i}$ Resolution of Group Membership}

Entities can form units of administration. We call them groups, although there are plenty of other names for this concept. It is especially applied by roles and domains, which are chiefly applied to subjects and objects. Actions, however, can also be grouped into, for example, EXIST(open, use, reset, assign, goto), CREATE(create, append, insert, add), READ(read, select, retrieve, request, list, display, report, print, copy), WRITE(write, alter, modify, update, change, replace, edit), EXECUTE(execute, start, run), and DESTROY(destroy, delete, remove). In such a way, more generic actions are achieved.

\section{Facts}

The facts are the memberships. A membership can be described explicitly by using an individual identity, or implicitly by giving a condition which is related to individual's properties. In the latter case, group facts consist obviously of rules and facts again.

\section{Rules}

The membership is a special relationship, which connects entities and groups. So, there is just one predefined rule: every entity $e$ gets the access rights of those groups $g$ where it is a member of, formally:

$$
\left(\mathfrak{a}_{g}, \mathrm{~A}_{\mathrm{S}}\right) \in \mathbb{R}_{\mathrm{IG} U \mathrm{I}} \wedge e \in g \Rightarrow\left(\mathfrak{a}_{e}, \mathrm{~A}_{\mathrm{S}}\right) \in \mathbb{R}_{1}
$$

Note that this group resolution is the basis of access rights.

\subsubsection{2il $\rightarrow 21 i_{\text {II }}$ Propagation between Entities}

Propagation can be defined between entities as well. Examples are right propagations from an inferior to a superior, or from a superobject to a subobject within object-oriented environments (we mention from a great variety just [JoDi93, JaKo90]). We have analogous rules and facts as given by the propagation between groups. So, we repeat just the general entity propagation rule:

$$
\left(\mathfrak{a}_{e^{\prime}}, A_{s}\right) \in \mathbb{R}_{1} \wedge\left(e^{\prime}, e\right) \in \Re \Rightarrow\left(\mathfrak{a}_{e}, A_{s}\right) \in \mathbb{R}_{[I]}
$$

\subsubsection{SUMMARY}

The idea of propagation is to exploit a relationship by using its semantics. So, access data consists of facts, describing the relationship, and rules, describing the propagation. Note that we use access rights $2 \pi$ in the sense of $2 \pi_{[I]}$ and that $2 \pi_{G, I} \equiv 2 \pi$ if groups and propagations would not be used. 


\subsection{Access Rules $\rightarrow \mathbb{R}_{\text {gur }}:$ Creating Initial Rights}

Access rules define initial rights both explicitly and implicitly by a consideration of entity properties. A particular right is given if entities have a relationship which is based on the properties of the particular entities. These entities have typically different entity types. A very clear example is the owner relationship which is defined between subjects and objects. If a subject owns an object, it is, following the ownership rule, able to perform any action on this object. However, the simple and the ${ }^{*}$-property of the multilevel security [BeLa73, Bell74, BeLa76] are examples, too. Finally, Minsky gave a nice example which we would like to repeat here:

"When a highway patrolman is sent to his duty, he has to be given the authority to cite traffic violators. This cannot be done explicitly for each violator because at the time that the patrolman is sent to his duty, the traffic violator does not exist, and the identity of the future violators is not known, so that it is impossible to construct individual access rights for the violators at that time. The point is that the patrolman's authority has to do with the behavior of motorists, not their identity." [Mins78]

\section{Facts}

The entity properties with respect to a particular relationship $\Re$ have to be managed.

\section{Rules}

An access rule describes the generation of access rights by consideration of properties of entities on an abstract level. This can be described formally as:

$$
\left(x_{1}, x_{2}, \ldots, x_{n}\right) \in \Re \Rightarrow\left(a_{\left\{x_{1}, x_{2} \ldots, x_{n}\right\}}, A_{S}\right) \in \mathbb{R}_{G U I}
$$

With other words, an access rule does:

select all accesses from $2 \mathbb{1}_{G \cup I}$ where $\left(x_{1}, x_{2}, \ldots, x_{n}\right) \in \Re$ (note the SQL syntax)

join this selection with one instance of specification attributes $A_{S}$ and append it to $2 R_{G U I}$

When considering $n$, we get four different cases:

n $=0$

$\left(\mathrm{x}_{1}, \mathrm{x}_{2}, \ldots, \mathrm{x}_{0}\right) \in \Re$ makes no sense and it means that there is no condition on the access. On the other hand, it holds that $\left\{\mathrm{x}_{1}, \mathrm{x}_{2}, \ldots, \mathrm{x}_{0}\right\} \equiv \varnothing$. Since $\varnothing \subseteq\{\mathrm{s}, \mathrm{a}, \mathrm{o}\}$ is always true, it holds that $\mathfrak{a}_{\varnothing} \equiv 2 \mathfrak{d}_{G U}$ and all accesses get the same specification attributes and are all members of $2 \pi_{\mathrm{GuI}}$. So, just one rule of this type is meaningful! A second rule is either contradictory or will never hold due to a lower priority.

Such a rule is suitable to describe a closure world assumption! To fulfill this task, this rule either has to have the lowest priority, which would ensure that this rule holds only if no other rule holds, or has only to be activated when the derivation process creates an empty access right set.

The (usually used) closed world assumption would be described as:

TRUE $\Rightarrow((s, a, o),(0,-)) \in Z_{G U}$ 
$\mathrm{n}=1$

$(x) \in \Re$ means a condition on one part of the access. This case is seldom used but could be useful to make a closure world assumption with respect to particular objects or to forbid the usage of a particular action.

Such a rule could be used to give a central authority all access rights:

$s=R O O T \Rightarrow\left(a_{i s, j},(1000,+)\right) \in 2 \pi_{G U}$

- $n=2$

$\left(\mathrm{x}_{1}, \mathrm{x}_{2}\right) \in \Re$ means a relationship between two parts of the access.

The ownership rule would be expressed as:

$s=$ o.owner $\Rightarrow\left(a_{i s, o j},(200,+)\right) \in 2 i_{G U}$

- $\mathrm{n}=3$

$\left(\mathrm{x}_{1}, \mathrm{x}_{2}, \mathrm{x}_{3}\right) \in \Re$ means a relationship between all three parts of an access. In the case that all parts are described by their identifier, we get the case of explicitly represented initial rights as the famous access matrix contains. So, tuples are the borderline case of an access rule of this type. However, $x_{i}$ could also be described by a (variable) property as, for example, a classification.

A rule where all three parts are involved could be used to give an advanced BLM property:

s.clearance $\geq 0$. classification $\wedge a \in$ o.read_interface $\Rightarrow\left(a_{\{s, a, 0,}(999,+)\right) \in 2 i_{G U}$

\subsection{Summary and Conclusion}

Roughly speaking, there are two major parts of the verification process: firstly, the access data is processed and, finally, the specification attributes are applied. If an access control model would use all access data concepts and the discussed attributes, the algorithm would be:

apply access rules

first of all, process the access rules and create initial rights

apply group propagation

derive more initial rights by propagation on the group level

apply group membership

resolve all group memberships and produce access rights

apply entity propagation

derive more access rights by propagation on the entity level

consider access

consider only the access rights which contain the requested access

apply predicates

select just the valid access rights

apply priority

select the access right with the highest priority

apply sign

declare the access as authorized or unauthorized depending on the sign

Figure 3 Verification Algorithm 
This algorithm should make clear that the distinction between explicit and implicit rights, which is often made in the literature (we note exemplary just [BeOS94]), makes no sense at all because there are no explicit access rights. Just tuples represent more or less explicit access rights by explicit initial rights, which are, however, the basis for three propagation steps! Therefore, those discussions should shift to discussions about different "types" of implicit access rights.

The ADF can be queried by the AEF as well as by another (foreign) system. The distinction between target and foreign systems can be made easily by a very small security kernel within the security module (described by a stroke-dotted line), which intercepts the query and checks the querying system against a list of 'permitted' systems. Note that this approach is based on the assumption that the identity of the querying system can be traced correctly, which has to be enforced by appropriate measures! This, however, is an implementation issue.

Summing up, an access control model has to specify which specification attributes and which concepts of access data are used. Based on these decisions, the verification algorithm has to be specified as well.

\section{Authorization}

Instances of concepts of access data are rules or facts and subject of protection. The administration of access data is known as authorization too. Authorization regulations are necessary to prevent that arbitrary access rights can be distributed to everybody. Without restrictions, access control would be meaningless because everybody could get every right.

Our following considerations are a repetition of everything, we have mentioned up to now; but on a higher level. So, we can shortcut our explanations and focus on additional aspects:

\subsection{Coal of Authorization}

Again, we have to distinguish between an Authorization Control Enforcement Facility (ACEF) and a Decision Facility (ACDF), a mapping between the activities within the target system (here: manipulations of access data) and authorizations, a verification algorithm, and regulations for the administration of the ACDF database. While the ACEF has to be built into the $\mathrm{ADF}$, which will not be considered further, the ACDF has to decide whether an authorization is authorized or not. In the following, an authorization is defined as:

Definition 5 Authorization

Within the process of an authorization we have to distinguish between:

the grantor which we call the authorization subject $\mathrm{s}_{\mathrm{a}}$,

the authorization mode $\mathrm{m}_{\mathrm{a}}$ and

the authorization object $\mathrm{o}_{\mathrm{a}}$.

Therefore, we get the definition of an authorization $\boldsymbol{a}$ as a triple:

Analogously, $\mathrm{A}$ is the set of all authorizations.

$$
\boldsymbol{a}=\left(\mathrm{s}_{\mathrm{a}}, \mathrm{m}_{\mathrm{a}}, \mathrm{o}_{\mathrm{a}}\right)
$$

\subsection{Mapping Between ADF Accesses and Authorizations}

The similarity with an access as defined earlier is intended. While an ACS protects a target (information) system, authorizations manipulate the objects of the ACS itself and have to be 
controlled as well. Note, however, that while accesses deal with entities of a target system, authorizations deal with subjects, actions, and objects of the ACS!

Authorization subjects have to be defined, where the relationship with the subjects of the target system can be arbitrarily chosen. If, however, these two subject sets are disjunct, the principle of Segregation of Duties is enforced. Note the necessity of an identification realization for authorization subjects!

The authorization objects are known; they are, as access data, either real or as rights, virtual. Although authorizations can be realized only on real objects, it has to be possible to specify an authorization on a virtual object as well and to count back. In particular, we have to deal with:

Real Authorization Objects

Real objects are access rules and their corresponding entity properties, propagation rules and the structures where the propagations are defined on, as well as group memberships.

Virtual Authorization Objects

Virtual objects are initial rights containing authorization groups, access rights, and the access status. By the way, we already dealt with an authorization restriction on access rights in Chapter 3.3.1, where we demanded that the priority of each new access right has to be unique and comparable with all other priorities of access rights with the same access.

Let us illustrate these relationships:

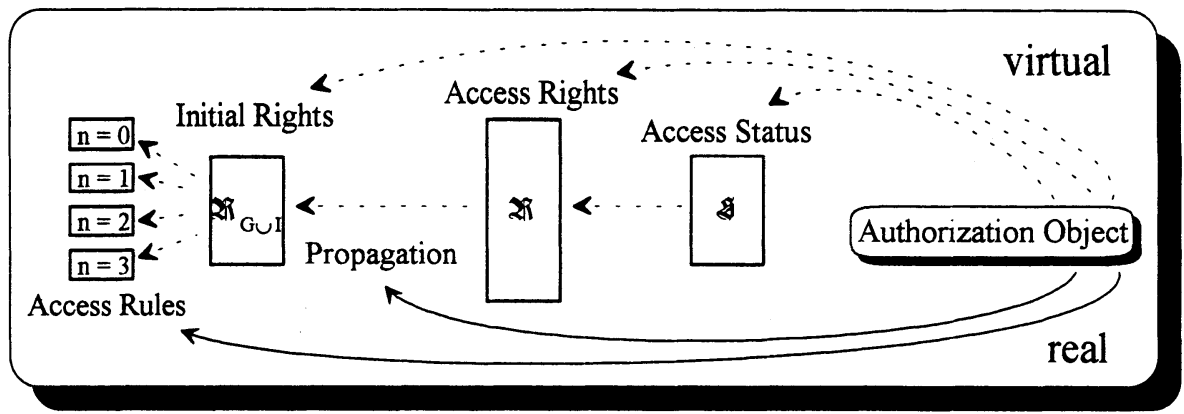

Figure 4 Authorization Objects and their Relationships

The discussion about virtual and real objects is concerned with the discussion about accesses which should be authorized (the goal: $\mathfrak{Z}$ and $\mathfrak{8}$ ) and which are authorized (the basis $\boldsymbol{g}$ ).

Keeping this quite complex structure in mind, we will abbreviate it in the remainder with:

$$
\mathfrak{g} \leftarrow \mathbb{2} \leftarrow \mathfrak{B}
$$

The authorization mode depends, of course, on the authorization object. In the literature, however, just two (generic) modes are considered: Grant and Revoke.

\subsection{Verification of Authorizations}

The ACDF has to define whether an authorization is authorized or not. The following definition provides a formal basis: 
Definition 6 Authorization Status

The authorization status is the set of all authorized authorizations and is denoted by $\mathbf{8}$.

Consequently, $\mathbf{\nabla} \subseteq \mathbf{A}$ holds. Let $\mathbb{Z}$ denote a set of authorization data. ACDF has, consequently, to provide the mapping $\rightarrow \rightarrow \mathbf{X}$. Compare with Chapter 2.3 ! In fact, we could repeat our descriptions of Chapter 3 , which were concerned with the functionality of the ADF. Let us just highlight the most important points:

\subsubsection{Authorization Rights}

Definition 7 Authorization Rights

An authorization right $r$ consists of an authorization $\mathfrak{a}$ and some further specification attributes, so $r=\left(\boldsymbol{a}, \mathrm{A}_{\mathrm{s}}{ }^{\prime}\right)$. All authorization rights are denoted by $\mathbb{q}$.

A usual assumption is a positive system of authorization rights with a closed world assumption. So, there is no need for additional specifications $\left(A_{s}^{\prime}=\varnothing\right)$ and $\bar{\nabla} \equiv \nexists$. However, we could imagine to extend this view $\left(A_{s}^{\prime} \neq \varnothing\right)$ ! In this case, a derivation algebra is needed to derive 8 from an.

If can be described explicitly (by enumeration) or implicitly (by rules). However, in the same way as access rights are represented by access data, authorization rights are described by authorization data 2 consisting of rules and facts.

\subsubsection{Authorization Data}

The same concepts as used for the access verification can be applied again. So, we deal with authorization groups, authorization propagations, and authorization rules. A central administrator would be one example for an authorization (subject) group. And we find the same taxonomy of authorization rules:

Authorization rules which are not related to a part of the triple $\left(\mathrm{s}_{\mathrm{a}}, \mathrm{m}_{\mathrm{a}}, \mathrm{o}_{\mathrm{a}}\right)$ :

With such rules very general statements are made. In the sense of a closure assumption for access rights, either all authorization can be permitted or forbidden unless they are specified explicitly.

Authorization rules dealing with one part of the triple:

The administration paradigm, for example, is based on an authorization rule of this kind: a (central) administrator may administrate everything for everybody. Such a rule, however, can be applied for particular objects or groups of objects as well.

Authorization rules dealing with two parts of the triple and their relationship:

The ownership paradigm is based on a rule of this type: only if a subject owns (whatever this means) an object, (s)he may administrate all access rights related to this object. We find here a relationship between the source subject and the ability. However, also other relationships can be used: subordination between source and target subject; the consideration whether the target subject already have the ability; etc.

Authorization rules dealing with all parts of the triple:

This means, in fact, to describe an authorization right explicitly and is the borderline case of a rule because just one derivation is possible.

In the following figure, we want to sketch this derivation process. Based on authorization data, authorization rights can be derived and based on this set, an authorization can be decided as 
authorized or not. Note that if the authorization object is of a virtual type, corresponding authorizations for authorization objects of a real type have to be derived.

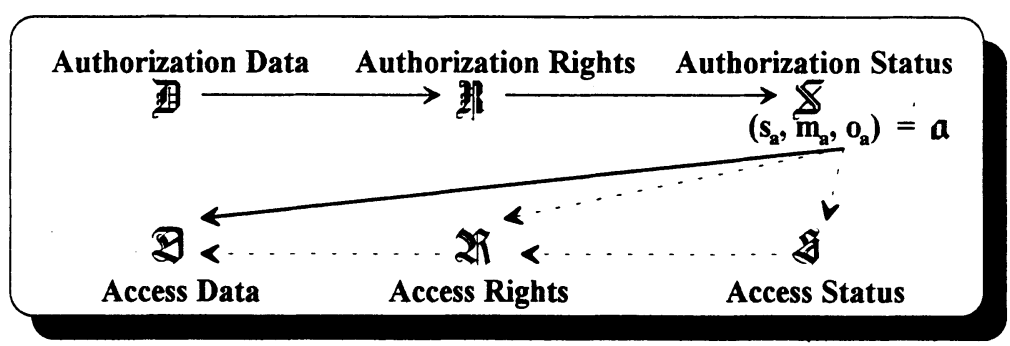

Figure 5 Structure of Authorization Verification

Note that this figure can be used as (an abbreviated) legend for our Gothic letters.

\subsection{Administration of Authorization Data}

The authorization data has to be administrated, again. So, we could repeat Chapter 4 up to here completely; but on one higher level. Instead of doing so, we give a more global view:

\section{Paradigms}

This spiral has to have an end; otherwise it would not be, of course, practical. So, there have to be some initial rights ruling everything. These rights will be called paradigm. To explain it, we need this general consideration:

\subsection{Authorization Layers}

There are obviously different authorization levels:

Zero Order Authorizations:

An access $\mathfrak{a}$ can be assumed as an authorization of order zero, the authorization object is here an object of the target system!

First Order Authorizations:

These authorizations describe rights to authorize an access as just explained. So, this concerns the definition of authorizations as given above, formally $\boldsymbol{a}=\left(s_{a}, m_{a}, \mathfrak{a}\right)$. Note that $a$ is, or course, virtual and has to be replaced by (real) access data.

* Second Order Authorizations:

These authorizations describe rights to authorize an authorization, formally $\boldsymbol{a}^{\prime}=\left(s_{\mathrm{a}}\right.$, $\left.\mathrm{m}_{\mathrm{a}}, \mathbf{a}\right)$. Although we call these administration rights 'authorizations' as well, they have other semantics. Here, the granted component $\boldsymbol{a}$ is not an access but an authorization $\left(s_{a}^{\prime}, m_{a}^{\prime}, \mathfrak{a}\right)$.

Higher Order Authorizations:

The administration of second order authorization and their administration leads to higher order authorizations. So, $\boldsymbol{a}^{\prime}$ has to be defined again by, for example, $\boldsymbol{a}^{\prime \prime}=\left(\mathrm{s}_{\mathrm{a}}\right.$, $\left.\mathrm{m}_{\mathrm{a}},\left(\mathrm{s}_{\mathrm{a}}^{\prime}, \mathrm{m}_{\mathrm{a}}^{\prime},\left(\mathrm{s}_{\mathrm{a}}^{\prime \prime}, \mathrm{m}_{\mathrm{a}}^{\prime \prime}, \mathrm{a}\right)\right)\right)$. 
Each order of authorization forms a layer and consists of a mapping between data and a status. The aim of layer $\mathrm{N}$ is to describe by its status the authorized administrations of data of layer $\mathrm{N}-1$. In order to do so, the authorization objects of layer $\mathrm{N}$ are elements of the verification process in layer $\mathrm{N}-1$. Authorizations on virtual authorization objects are here to be transformed to authorizations on real objects, which is illustrated by " $\leftarrow$ " in Figure 6.

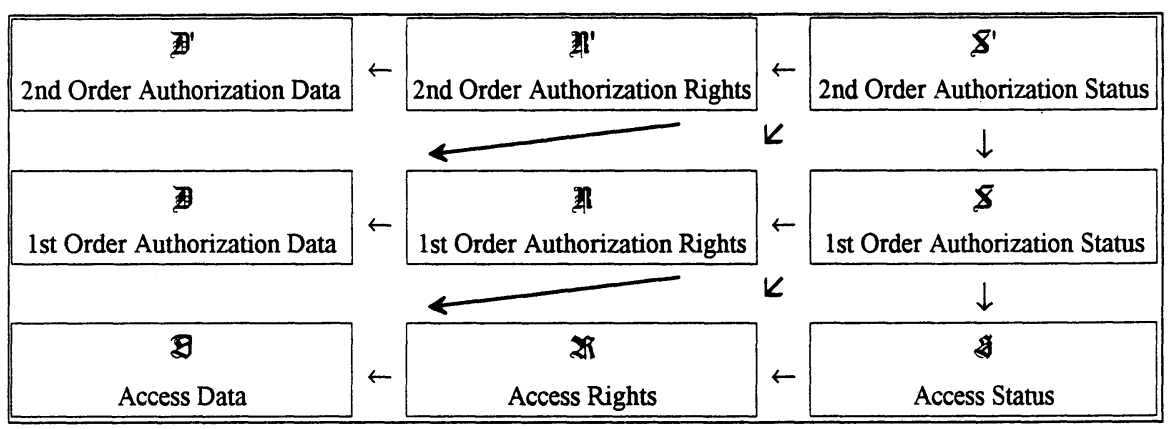

Figure 6 Authorizations on Various Levels

Every layer forms a logical unit, but they can be implemented together sharing the same ACEF.

\subsection{Definition}

There has to be some initial authorization data, being the basis for initial access rights. Otherwise, everybody can receive every access right (see for the Safety Problem Harrison, Ruzzo, and Ullman [HaRU76]): This data is defined by a paradigm:

Definition 8 Paradigm

A paradigm realizes the chosen security policy with respect to access control by initial rights. It consists of a (mandatory) set of authorization data; maybe on any level, so on layer 0 (access layer) as well. There is, however, one boundery condition: there are authorization rules at least in the highest layer.

These initial rights form, with other words, a frame for all future access and authorization activities by an initial exclusion of rights. A paradigm, however, is decisive for an access control model as the following chapter shows.

\subsection{Discretionary vs. Mandatory Access Control}

Two access controls are of public interest: Discretionary and Mandatory Access Control. According to the Orange Book [DoD85], they are defined by "A means of restricting access to objects based on:

Discretionary Access Control

the identity of subjects and/or groups to which they belong. The controls are discretionary in the sense that a subject with a certain access permission is capable of passing those permissions (perhaps indirectly) on to any other subject."

Mandatory Access Control

the sensitivity (as represented by a label) of the information contained in the objects and the formal authorization (i.e. clearance) of the subjects to access information of 
such sensitivity. Mandatory controls are driven by the results of a comparison between the user's trust level or clearance and the sensitivity of the information."

Both definitions provide merely a hazy idea. While the first one aims on the authorization aspect, the second is concerned with the verification aspect. Let us describe these controls in terms of our framework

\subsubsection{Discretionary Access Control}

In the following figure, we sketch DAC. We recommend to read the figure bottom-up, starting with the access layer:

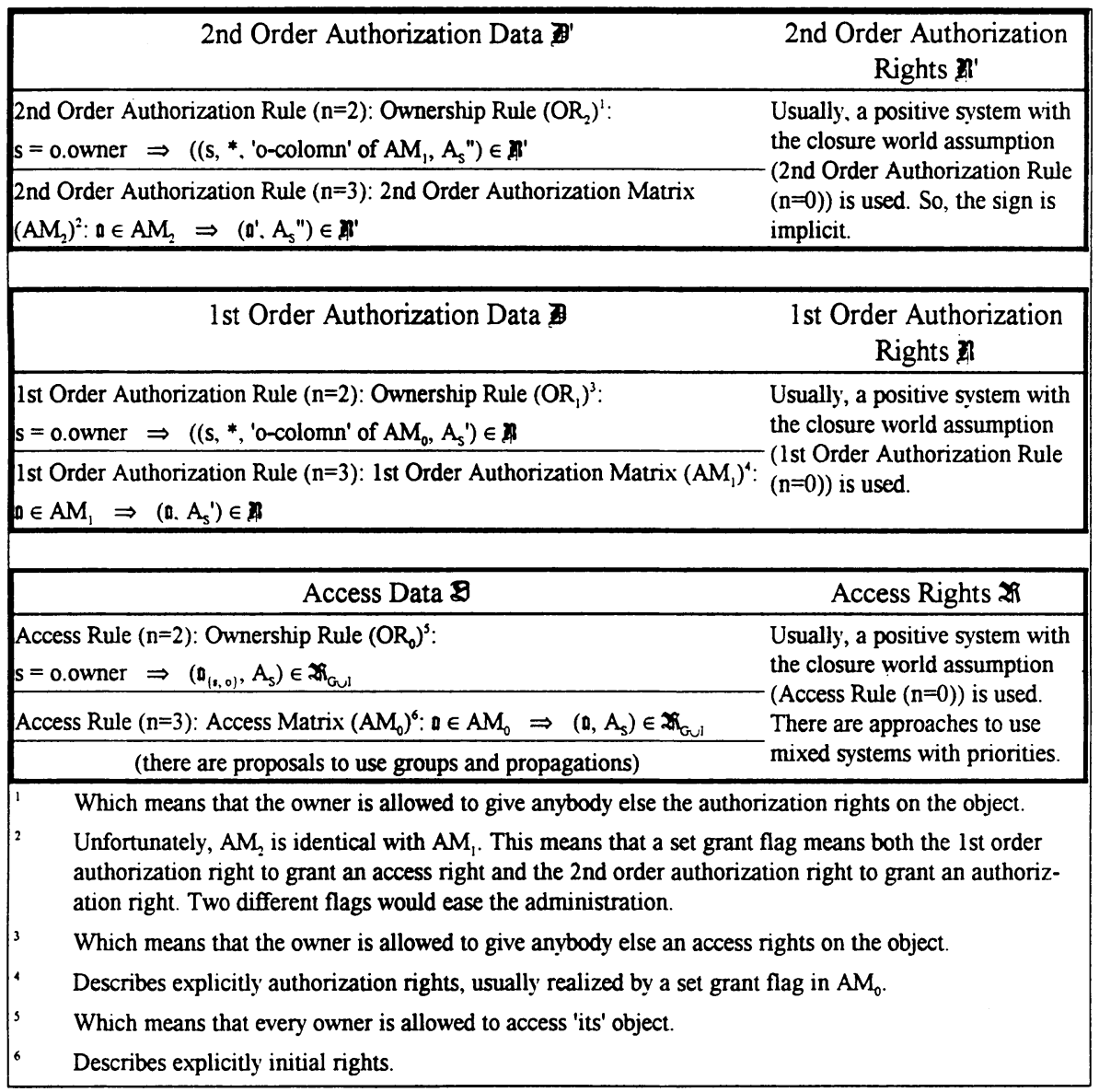

Figure 7 DAC Layers

The most simplest Ownership Paradigm consists of the ownership rules $\mathrm{OR}_{0}$ and $\mathrm{OR}_{1}$ describing basically the verification and authorization aspect. However, often $\mathrm{OR}_{2}$ is added to manage grant flags. Note that $\mathrm{AM}_{0}$ and $\mathrm{AM}_{1}\left(\equiv \mathrm{AM}_{2}\right)$ comes dynamically into existence. 


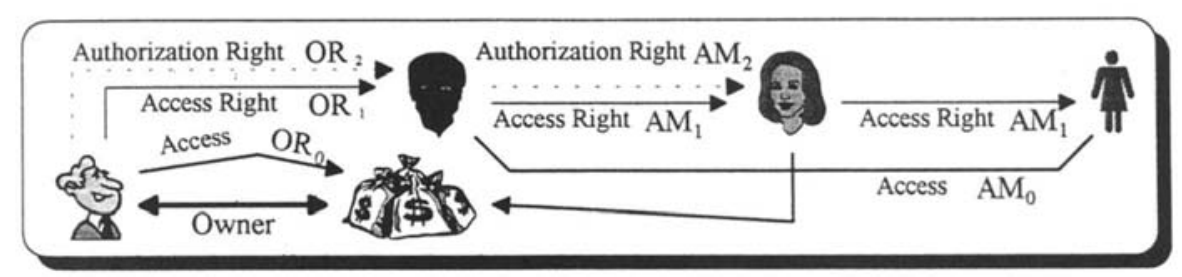

Figure 8 Ownership Rules and Access / Authorization Matrixes

In Figure 8, we sketched possible actions under the Ownership Paradigm together with the reason why these actions are allowed. Note that the authorizations in layer $\mathrm{N}$ are related to the allowed activities in layer $\mathrm{N}-1$.

A possible enhancement of this paradigm is concerned with central administrated prohibitions to receive access rights. In our figure, we would have to add:

\begin{tabular}{|c|}
\hline 2nd Order Authorization Data $\mathbb{Z}^{\prime}$ \\
\hline $\begin{array}{l}\text { 2nd Order Authorization Rule }(\mathrm{n}=0) \text { : Administrator Determination: } \\
\text { TRUE } \Rightarrow\left(\left(\mathrm{s}, * \text {, membership of 'central_Administrator', } \mathrm{A}_{\mathrm{s}}{ }^{\prime}\right) \in \mathrm{N}^{\prime}\right.\end{array}$ \\
\hline 1st Order Authorization Data $\nexists$ \\
\hline $\begin{array}{l}\text { 1st Order Authorization Rule }(\mathrm{n}=1) \text { : Administrator Rule: } \\
\mathrm{s}=\text { 'central_Administrator' } \Rightarrow\left(\left(\mathrm{s}, *,-\mathrm{AM}_{0}, \mathrm{~A}_{\mathrm{s}}{ }^{\prime}\right) \in \mathbb{n}\right.\end{array}$ \\
\hline 'central_Administrator' is a group \\
\hline Access Data 8 \\
\hline Access Rule $(n=3)$ : Access Matrix $\left(-\mathrm{AM}_{0}\right)^{1}: \mathfrak{a} \in-\mathrm{AM}_{0} \Rightarrow\left(\mathfrak{a}, \mathrm{A}_{\mathrm{s}}\right) \in \mathbb{A}_{\mathrm{Cul}}$ \\
\hline $1 \quad$ Describes explicitly negative access rights, which take implicitly priority over permissions in $\mathrm{AM}_{0}$ \\
\hline
\end{tabular}

Figure 9 Advanced DAC Description

\subsubsection{Mandatory Access Control}

Mandatory access control is based on the administrator paradigm and its description is much more simple: 


\begin{tabular}{|c|}
\hline 2nd Order Authorization Data $\nexists^{\prime}$ \\
\hline $\begin{array}{l}\text { 2nd Order Authorization Rule }(\mathrm{n}=0) \text { : Administrator Determination: } \\
\text { TRUE } \Rightarrow\left(\left(\mathrm{s} .{ }^{*} \text {. membership of 'central_Administrator', } \mathrm{A}_{\mathrm{s}}\right)^{\prime}\right) \in \mathrm{n}^{\prime}\end{array}$ \\
\hline 1st Order Authorization Data \\
\hline 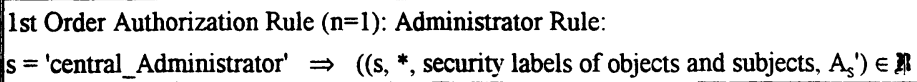 \\
\hline 'central_Administrator' is a subject group \\
\hline Access Data 2 \\
\hline $\begin{array}{l}\text { Access Rule }(n=3) \text { : simple security property: } \\
\text { s.clearance } \geq 0 \text {.classification } \wedge \mathrm{a}=\mathrm{read} \Rightarrow\left((\mathrm{s}, \mathrm{a}, 0), \mathrm{A}_{\mathrm{s}}\right) \in 2 \pi_{\mathrm{Gul}}\end{array}$ \\
\hline $\begin{array}{l}\text { Access Rule }(n=3) \text { : star security property: } \\
\text { s.clearance } \leq \text { o.classification } \wedge \mathrm{a}=\text { write } \Rightarrow\left((s, a, 0), A_{s}\right) \in 2 \pi_{\text {Gul }}\end{array}$ \\
\hline
\end{tabular}

Figure 10 MAC Description

Mandatory Access Control is completely described by

- two access rules embodying the simple and the star $(*)$ security property, which are defined on subject and object attributes,

- one 1st order authorization rule determining the responsibility of a central administrator for the security label, and

- one 2 nd order authorization rule defining who may act in the role of the central administrator. (It is also possible to define the administrator not by a group but directly.)

- A positive system with a closed world assumption on all layers.

\section{Summary and Conclusion}

We have described a framework for access control models, which defines the requirements on such a model. In particular, a model has to include:

- A discussion about the mapping between the elements of the target system and the entities of an access, which were subjects, actions, and objects. This mapping is necessary to allow a mapping between the activities within the target system and an access.

- The realization of a paradigm by initial data on various layers. By this, also the used layers are defined.

- The definition of the verification algorithm within each layer. Based on data, rights have to be derived which form the basis for the derivation of the status. While each authorization status rules the administration activities of the underlying layer, the access status rules over the activities within the target system. Note that the structure of each layer is the same.

Finally, we have shown a description of DAC and MAC in terms of our framework, although we did not consider particular target systems as relational database systems or operating systems. Such a target system requires additional considerations about its structure and elements.

This framework has shown to be very powerful during a study on existing access control literature (included in [Lau95b]). Each reference was exactly in place. On the other hand, it has 
shown its ability to create new models as well. In [Lau94, Lau95b], we described an enterprise model, which is based on hierarchies. Especially, the effect of propagation on authorization was investigated.

\section{REFERENCES}

[AbSm91] Marshall D. Abrams, Gary W. Smith:

"A Generalized Framework for Database Access Controls"

Database Security IV: Status and Prospects, eds. S. Jajodia and C.E. Landwehr, pp. 171-178, North-Holland, 1991.

[AbO192] Marshall D. Abrams, Ingrid M. Olson:

"Rule-Based Trusted Access Control"

IT Security: The Need for International Cooperation, eds. G.G. Gable and W.J. Caelli, pp. 409-420, Elsevier Science Publishers B.V. (North-Holland), 1992.

[BeLa73] D. Elliott Bell, Leonard J. LaPadula:

"Secure Computer Systems: Mathematical Foundations"

[MTR-2547| ESD-TR-73-278, (Vol. I\&I)], The MIRE Corporation, Bedford, Massachusetts, 33 p., November 1973.

[Bell74] D. Elliott Bell:

"Secure Computer Systems: A Refinement on the Mathematical Model"

[MTR-2547 | ESD-TR-73-278, (Vol.3)], The MITRE Corporation, Bedford, Massachusetts, 31 p., April 1974.

[BeLa76] D. Elliott Bell, Leonard J. LaPadula:

"Secure Computer Systems: Unified Exposition and Multics Interpretation"

[MTR-2997 | ESD-TR-75-306], The MITRE Corporation, Bedford, Massachusetts, March 1976.

[BeOS94] E. Bertino, F. Origgi, P. Samarati:

"A New Authorization Model for Object-Oriented Databases"

in: Proc. of the IFIP WG 11.3 8th Annual Working Conference on Database Security, 1994.

[Brüg92] Hans $\mathrm{H}$. Brüggemann:

"Rights in an Object-Oriented Environment"

Database Security V: Status and Prospects, eds. C.E. Landwehr and S. Jajodia, pp. 99-115, North-Holland, 1992.

[ClWi87] David D. Clark, David R. Wilson:

"A Comparsion of Commercial and Military Computer Security Policies"

Proc. IEEE Symposium on Security and Privacy, IEEE Computer Society, pp. 184-194, Oakland, CA, April 1987.

[DoD85] Department of Defence

"Trusted Computer System Evaluation Criteria" (The Orange Book)

Department of Defence, National Computer Security Center, December 1985. DoD 5200.28-STD

[HaRU76] M.A. Harrison, W.L. Ruzzo, J.D. Ullman:

"Protection in Operating Systems"

Communications of the ACM, vol.19(8), pp. 461-471, 1976.

[ISO91] International Standard Organization:

"Security Frameworks Part 3: Access Control"

ISO/ECC CD 10181-3, 1991.

[JaKo90] Sushil Jajodia, B. Kogan:

"Integrating an Object-Oriented Data Model with Multilevel Security"

Tech. Rapport RADC-TC-90-91, Rome Air Development Center, Griffiss AFB, NY, 1990.

[JaSa90] Sushil Jajodia, Ravi Sandhu:

"Polyinstantiation Integrity in Multilevel Relations"

IEEE Symposium on Security and Privacy, pp. 104-115, Oakland, California, May 1990. 
[JaSa91] Sushil Jajodia, Ravi Sandhu:

"Toward a Multilevel Secure Relational Data Model"

Proc. ACM SIGMOD Conf., Denver, Colorado, 1991.

[JoDi93] Dirk Jonscher, Klaus Dittrich:

"A Formal Security Model Based on an Object-Oriented Data Model"

Institut für Informatik der Universität Zürich, Internal Report no.93.41, 51 p., Nov. 1993.

[Lau94] Burkhard Lau:

"A Decentralized Approach for Authorization"

IT Security: The Need for International Cooperation, ed. W.J. Caelli, pp. 441-462, North-Holland, 1994.

[Lau95a] Burkhard Lau

"Towards a Comprehensive View on Security"

IT-Security'94, Post-Workshop Proceedings, G. Pernul (ed.), R. Oldenbourg Wien München, Jan. 1995.

[Lau95b] Burkhard Lau

"Access Control: Concepts and Applications"

Ph.D. Thesis, Delft University of Technology, Department of Information Systems, Aug. 1995.

[Lubi92] Astrid Lubinski:

"Zugriffsschutz in Unternehmen"

Rostocker Informatikberichte 7/92, FB Informatik, Universităt Rostock, 31 p., 1992.

[Lubi93] Astrid Lubinski:

"Ein Rollen-Normen-Modell für den konzeptionellen Entwurf von Sicherheitsanforderungen in Unternehmens-Informationssystemen"

Proc. VIS'93 in: DuD Fachbeiträge 16, eds. G. Weck and P. Horster, pp. 67-84, 1993.

[Lunt89] Teresa F. Lunt:

"Access Control Policies for Database Systems"

Database Security, II: Status and Prospects, ed. Carl E. Landwehr, pp. 41-52, North-Holland, 1989.

[Mins78] Naftaly H. Minsky:

"An Operation-Control Scheme for Authorization in Computer Systems"

International Journal of Computer and Information Sciences, vol. 7, no 2, pp.157-191, 1978.

[MoDe92] Philip Morris, John A. McDermid:

"The structure of permissions: a normative framework for access rights"

Database Security V: Status and Prospects, eds. C.E. Landwehr and S. Jajodia, pp. 77-97, North-Holland, 1992

[RaWK88] F. Rabitti, D. Woelk, W. Kim:

"A Model of Authorization for Object-Oriented and Semantic Databases"

Proc. of the Int. Conf. on Extending Database Technology, Venice, Italy, pp. 231-250, March 1988.

[RBKW91] F. Rabitti, E. Bertino, W. Kim, D. Woelk:

"A Model of Authorization for Next-Generation Database Systems"

ACM Transactions on Database Systems, Vol. 16, No 1, S. 88 -131, March 1991. 